\title{
sciendo
}

\section{Tumor-induced osteomalacia - a mystery illness beyond aches, pains, and depression}

\author{
Huajing (Jing) Ni ${ }^{1,2}$, Roderick Clifton-BLIGH ${ }^{3,4}$, Malgorzata Monika BRzozowskA ${ }^{1,2}$ \\ ${ }^{1}$ Endocrinology Department, Sutherland Hospital, Sydney, NSW, Australia; ${ }^{2}$ Faculty of Medicine, University of New \\ South Wales, NSW, Australia; ${ }^{3}$ Endocrinology Department, Royal North Shore Hospital, Sydney, NSW, Australia; \\ ${ }^{4}$ Faculty of Medicine, University of Sydney, Australia \\ E-mail:m.brzozowska@unsw.edu.au
}

Objective. Tumor-induced osteomalacia (TIO) is a rare paraneoplastic syndrome characterized by chronic hypophosphatemia and osteomalacia. We present case of a patient with a protracted clinical course of TIO. TIO profoundly affected every aspect of his life with subsequent profound physical and psychosocial disabilities.

Method. The review of a complex clinical presentation, serial laboratory investigations, and imaging modalities of a patient with TIO caused by a mesenchymal tumor.

Results. The patient presented with chronic lower back pain, severe bilateral leg weakness, and multiple pathological fractures due to severe osteoporosis. His investigations revealed hypophosphatemia, low 1,25 dihydroxyvitamin D, phosphaturia and normal serum calcium, and parathyroid hormone. Elevated fibroblast growth factor 23 (FGF23) confirmed the diagnosis of TIO and ${ }^{68} \mathrm{Ga}$-DOTATATE-positron emission tomography/computed tomography (PET/CT) imaging correctly identified a tumor in the left femoral head. His clinical features and biochemical abnormalities promptly recovered after successful surgical resection of the mesenchymal tumor.

Conclusion. The present case demonstrated the need to extensively investigate causes of generalized bone pain in patients with hypophosphatemia, as TIO is highly curable. Importantly, ${ }^{68} \mathrm{Ga}-$ DOTATATE PET/CT imaging successfully identified the FGF23 producing tumor, which was undetectable by conventional imaging, favoring its early use in suspected TIO presentation. The present report highlights the importance of timely diagnosis of this complex medical condition, aiming to improve general awareness and enable better clinical outcomes for this rare disorder.

Key words: tumor-induced osteomalacia, oncogenic osteomalacia, paraneoplastic hypophosphatemia, mesenchymal tumor, phosphatonin, fibroblast growth factor-23

Tumor-induced osteomalacia (TIO), previously known as oncogenic osteomalacia, is a rare paraneoplastic metabolic bone disorder characterized by renal phosphate wasting, hypophosphatemia and osteomalacia (Carpenter 2003; Hu et al. 2016). This complex paraneoplastic syndrome was first described in 1947 by McCance with less than 500 reported cases to date (Liu et al. 2019). Lack of awareness amongst clinicians often leads to substantial delay in diagnosis of this rare condition with significant implications for affected individuals.

The pathogenesis of TIO is related to uncontrolled secretion of fibroblast growth factor 23 (FGF23) by mesenchymal tumor tissues. FGF23 regulates vitamin $\mathrm{D}$ and phosphate metabolism as it inhibits phosphate reabsorption at the proximal tubule, promoting renal

Corresponding author: Dr. Malgorzata Monika Brzozowska, Endocrinology Department, Sutherland Hospital, Sydney, NSW, Australia; Faculty of Medicine, University of New South Wales, Kensington, NSW, Australia; phone: (+61) 425335465; fax: (+61) 29540 8067; e-mail: m.brzozowska@unsw.edu.au, mbrzozowska@hotmail.com. 
phosphate excretion and inhibiting calcitriol production (Perwad et al. 2007). Low serum phosphate is physiologically accompanied by suppressed FGF23; consequently, finding of elevated serum FGF23 in hypophosphatemia strongly suggests either TIO (Chong et al. 2011) or an hereditary syndrome such as X-linked hypophosphatemic rickets (XLH), autosomal dominant (ADHR) and autosomal recessive (ARHR) hypophosphatemic rickets (Yamazaki et al. 2002; Jonsson et al. 2003).

We present a patient with TIO in whom delayed diagnosis was accompanied by serious physical and psychosocial disabilities that have significantly improved after surgical resection of causative tumor. We highlight the importance of timely diagnosis of this rare medical condition with the aim of improving the general awareness of this rare disorder.

\section{Subject and results}

A 48-year-old man presented with an acute exacerbation of chronic lower back pain and severe bilateral leg weakness, present for at least 6 years. Pain severely restricted his mobility confining him to life in his apartment. He was of short stature with height $155 \mathrm{~cm}$, weight of $80 \mathrm{~kg}$ and BMI of 33. Neurological examination revealed predominantly proximal muscle weakness in both upper and both lower limbs and associated muscle wasting. Whole spine magnetic resonance imaging (MRI) demonstrated mild disc disease without any obvious thoracolumbar spine fractures or enhancing lesions. Nerve conduction studies (2017) showed no evidence of generalized large fiber neuropathy or demyelinating features, and without clear neurogenic or myopathic features in the muscles sampled. Screens for vasculitis and multiple myeloma were negative.

Biochemistry found hypophosphatemia $[0.21-0.76 \mathrm{mmol} / \mathrm{L}$, reference range (RR) 0.75 $1.50 \mathrm{mmol} / \mathrm{L})]$ and despite intravenous and oral phosphate supplementations (up to $1 \mathrm{~g} /$ day) his phosphate values did not revert to the normal reference range. Serum calcium level remained within normal range. He had consistently raised alkaline phosphatase (ALP) between 175-347 U/L (RR 30-110 U/L), elevated intact parathyroid hormone (iPTH) up to $27.4 \mathrm{pmol} / \mathrm{L}$ (RR 1.6-6.9 pmol/L) and low 1,25-dihydroxyvitamin D [1,25(OH)2D] of $42 \mathrm{nmol} / \mathrm{L}$ (RR $60-200 \mathrm{nmol} / \mathrm{L}$ ) which improved over time with calcitriol supplementation (up to $1.25 \mu \mathrm{g}$ per day) (Table 1). Estimated maximum tubular reabsorption of phosphorus corrected for glomerular filtration rate (TmP/GFR) revealed reduced fractional reabsorption of phosphate of $0.433 \mathrm{mmol} / \mathrm{L}$ (RR $0.9-1.35 \mathrm{mmol} / \mathrm{L}$ for male aged years 45-55), consistent with renal phosphate wasting (Jan de Beur 2005).

A whole-body bone scan revealed thoracolumbar scoliosis and lower limb arthritis. Bone mineral density (BMD) measured by Dual Energy X-ray Absorptiometry (DXA) was severely low with lumbar spine (LS) T score of -4.6 , femoral neck (FN) T score of -2.9 , and total hip (TH) T score of -4.1 .

The patient continued to experience exacerbations of his back pain, not alleviated by CT guided steroid injections, as well pain radiating to his knees, shoulder and chest. Pelvic CT, skeletal survey and

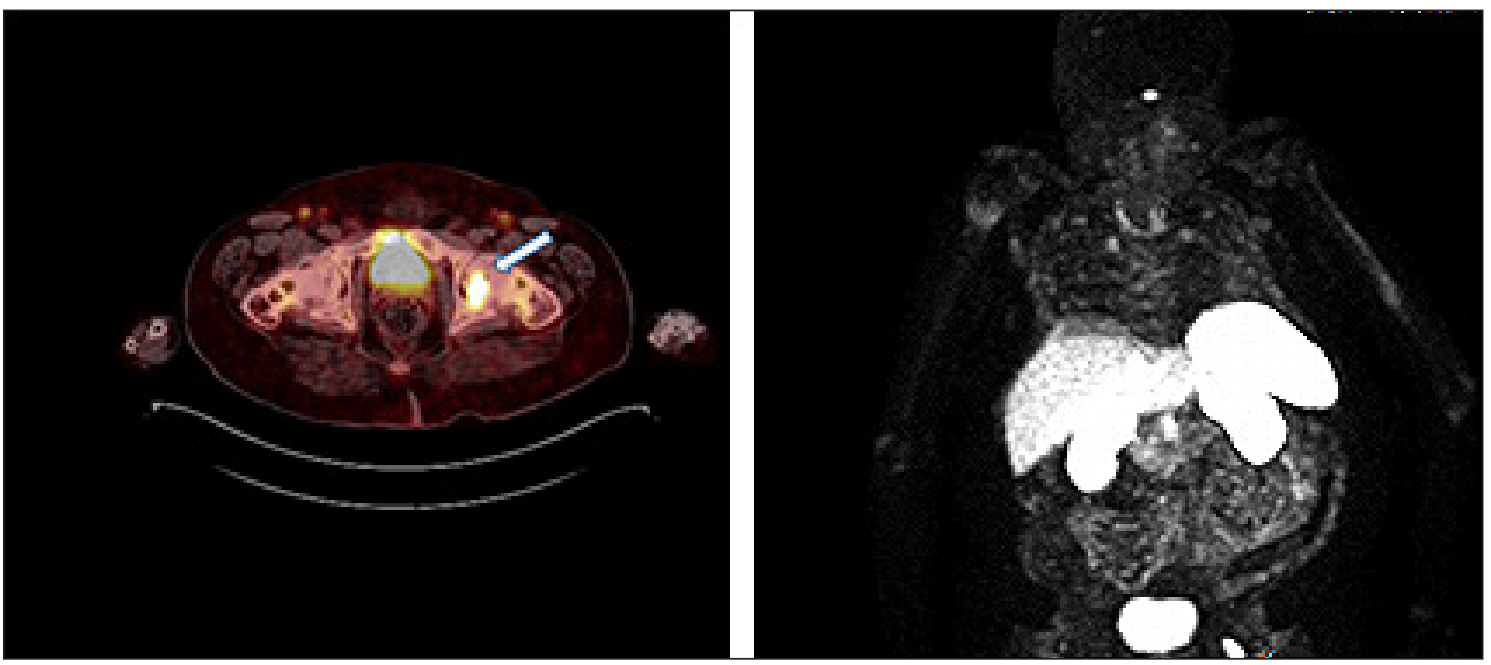

Figure 1. 68Ga-DOTATATE ([68Ga-DOTA0-Ty3] octreotate) PET/CT scan demonstrates a single abnormal uptake in the left femoral head. 
Table 1

Results of patient's laboratory tests over time

\begin{tabular}{|c|c|c|c|c|c|c|c|}
\hline \multirow[b]{2}{*}{ Parameters (ranges) } & \multicolumn{5}{|c|}{ Preoperative values } & \multicolumn{2}{|c|}{ Postoperative values } \\
\hline & $\begin{array}{c}20 / 06 / 17 \\
- \\
04 / 07 / 17 \\
\end{array}$ & $\begin{array}{c}31 / 12 / 17 \\
- \\
16 / 01 / 18 \\
\end{array}$ & $\begin{array}{c}16 / 02 / 18 \\
- \\
17 / 02 / 18 \\
\end{array}$ & $\begin{array}{c}31 / 03 / 18 \\
- \\
31 / 03 / 18 \\
\end{array}$ & $\begin{array}{c}27 / 09 / 19 \\
- \\
11 / 11 / 19 \\
\end{array}$ & $\begin{array}{c}09 / 12 / 19 \\
- \\
14 / 07 / 20 \\
\end{array}$ & $\begin{array}{c}\text { Reference } \\
\text { interval }\end{array}$ \\
\hline $\mathrm{Na}(\mathrm{mmol} / \mathrm{L})$ & $136-142$ & $137-142$ & $141-143$ & 144 & $139-145$ & $139-143$ & $135-145$ \\
\hline $\mathrm{K}(\mathrm{mmol} / \mathrm{L})$ & $3.7-4.9$ & 5.1 & $4.6-4.7$ & 4.8 & $4.0-5.1$ & $4.8-5.4$ & $3.5-5.2$ \\
\hline Bicarbonate $(\mathrm{mmol} / \mathrm{L})$ & $23-26$ & $25-28$ & $22-25$ & 25 & $25-30$ & $23-29$ & $22-32$ \\
\hline Creatinine $(\mu \mathrm{mol} / \mathrm{L})$ & $69-86$ & $74-76$ & $70-77$ & 72 & $60-69$ & $56-66$ & $60-110$ \\
\hline eGFR (mL/min) & $>90$ & $>90$ & $>90$ & $>90$ & $>90$ & $>90$ & $>90$ \\
\hline $\mathrm{Ca}(\mathrm{mmol} / \mathrm{L})$ & $2.14-2.41$ & $2.39-2.44$ & $2.05-2.13$ & 2.37 & $2.28-2.36$ & $2.32-2.48$ & $2.10-2.60$ \\
\hline Corrected Ca $(\mathrm{mmol} / \mathrm{L})$ & $2.16-2.34$ & $2.41-2.44$ & $2.10-2.16$ & 2.34 & $2.36-2.38$ & $2.32-2.44$ & $2.10-2.60$ \\
\hline $\mathrm{Mg}(\mathrm{mmol} / \mathrm{L})$ & $8.82-1.06$ & 0.80 & $0.77-0.79$ & 0.78 & $0.77-0.83$ & $0.73-0.89$ & $0.7-1.1$ \\
\hline $\mathrm{PO} 4(\mathrm{mmol} / \mathrm{L})$ & $0.34-0.68$ & $0.49-0.60$ & $0.24-0.69$ & 0.21 & $0.49-0.76$ & $1.32-1.66$ & $0.75-1.50$ \\
\hline iPTH (pmol/L) & 27.4 & 9.9 & l & I & 8.1 & $5.5-18.6$ & $1.6-6.9$ \\
\hline FGF23 (pg/ml) & & & & & 205 & $12.7-43.1$ & $<50$ \\
\hline HbAlc (\%) & 1 & 5.9 & I & l & l & l & $4.0-6.0$ \\
\hline $25(\mathrm{OH}) \mathrm{D}(\mathrm{nmol} / \mathrm{L})$ & l & 58 & l & l & $41-53$ & $70-77$ & $51-250$ \\
\hline $1,25(\mathrm{OH})_{2} \mathrm{D}(\mathrm{nmol} / \mathrm{L})$ & & 42 & & & 62 & 95 & $60-200$ \\
\hline Albumin $(\mathrm{g} / \mathrm{L})$ & $33-41$ & $35-38$ & $33-34$ & l & $31-37$ & $34-40$ & $33-48$ \\
\hline Alanine aminotransferase $(\mathrm{U} / \mathrm{L})$ & 31 & 38 & l & I & $5-17$ & l & $<45$ \\
\hline Aspartate aminotransferase (U/L) & $26-28$ & 36 & I & l & $11-21$ & l & $<45$ \\
\hline Alkaline phosphatase (U/L) & $147-175$ & 347 & l & l & 347 & 283 & $30-110$ \\
\hline$\gamma$-Glutamyl transferase (U/L) & $92-124$ & 166 & l & l & $34-57$ & l & $0-50$ \\
\hline Bilirubin $(\mu \mathrm{mol} / \mathrm{L})$ & $7-9$ & 8 & l & 1 & $3-6$ & 1 & $0-25$ \\
\hline Creatinine Kinase (U/L) & $42-63$ & l & l & l & l & l & $<200$ \\
\hline Free T4 (pmol/L) & 1 & 17.6 & 1 & I & l & 1 & $12.0-22.0$ \\
\hline Free T3 $(\mathrm{pmol} / \mathrm{L})$ & 1 & 5.0 & l & l & l & 1 & $3.10-6.80$ \\
\hline TSH (mIU/L) & 1 & 0.6 & l & l & 0.7 & l & $0.27-4.20$ \\
\hline ACTH (ng/mL) & 17.7 & $3.9-6.7$ & l & l & 12.8 & $7.2-14.7$ & $7.2-63.3$ \\
\hline Cortisol (nmol/L) & 312 & $64-638$ & 1 & 1 & 487 & 1 & $166-507$ \\
\hline $24 \mathrm{~h}$ urine free cortisol (nmol/day) & & 30 & & & & & $<166$ \\
\hline Testosterone (nmol/L) & 11.4 & 6.5 & 1 & I & 8.0 & 1 & $10.4-30.9$ \\
\hline Estradiol (pmol/L) & 1 & $71-78$ & l & l & l & & $36-155$ \\
\hline DHEAS $(\mu \mathrm{mol} / \mathrm{L})$ & 1 & $<2.1$ & 1 & l & l & 1 & $4.5-48$ \\
\hline $\mathrm{LH}(\mathrm{mIU} / \mathrm{ml})$ & 8.2 & 2.5 & l & l & 2.7 & l & $1.5-12.4$ \\
\hline FSH $(\mathrm{mIU} / \mathrm{L})$ & 2.6 & 3.3 & 1 & I & 2.2 & 1 & $1.7-8.6$ \\
\hline Prolactin $(\mathrm{mU} / \mathrm{L})$ & 1 & $477-512$ & l & l & 391 & 1 & $86-324$ \\
\hline IGF1 (nmol/L) & I & 28 & 1 & I & 19 & 1 & $10.0-32.5$ \\
\hline Hemoglobin $(g / L)$ & $132-153$ & $130-147$ & 130 & 146 & $115-127$ & $113-121$ & $130-180$ \\
\hline Platelets $\left(\times 10^{9} / \mathrm{L}\right)$ & $303-374$ & 257-309 & 274 & 276 & 276-334 & $310-669$ & $150-450$ \\
\hline
\end{tabular}

Abbreviations: $\mathrm{Na}$ - sodium; $\mathrm{K}$ - potassium; eGFR - estimated glomerular filtration rate; $\mathrm{Ca}$ - calcium, $\mathrm{Mg}$ - magnesium; PO4 - phosphorous; iPTH - intact parathyroid hormone; FGF23 - fibroblast growth factor 23; HbA1c - hemoglobin A1c; 25(OH)D 25-hydroxyvitamin D; 1,25(OH)2D - 1,25-dihydroxyvitamin D; T4 - thyroxine; T3 - triiodothyronine; TSH - thyroid stimulating hormone; ACTH - adrenocorticotropic hormone; DHEAS - dehydroepiandrosterone sulfate; LH - luteinizing hormone; FSH follicle-stimulating hormone; IGF1 - insulin-like growth factor 1. 
repeat lumbar spine MRI showed no evidence of any lytic lesions or fractures. Over the next 18 months the patient suffered from minimal trauma fractures involving his bilateral clavicles, multiple ribs, right humeral neck and left humeral midshaft. After two years, a repeat DXA scan showed decrease in bone density with lumbar spine (LS) T score of -5.2 and total hip (TH) T score of -3.5 despite calcitriol $(0.5 \mu \mathrm{g}$ twice daily), cholecalciferol (50 $\mu$ g daily) and a trial of denosumab. Review of his hormonal assays revealed hypogonadotropic hypogonadism without evidence of an additional endocrinopathy (Table 1). No pituitary pathology was found on MRI.

The patient continued to find phosphate replacement challenging, due to ongoing diarrhea, hence the persistent hypophosphatemia (Table 1). Finally, his progressive physical disability led to financial crisis when he lost ownership of his home.

${ }^{68} \mathrm{Ga}$-DOTATATE ([68Ga-DOTA0-Ty3] octreotate) positron emission tomography/computed tomography (PET/CT) scan showed a single focus of significant abnormal uptake in the left femoral head (Figure 1). At this time, serum intact FGF23 was elevated at $205 \mathrm{pg} / \mathrm{mL}$ (DiaSorin Liaison, RR $<50 \mathrm{pg} / \mathrm{mL}$ ). MRI revealed bilateral non-acute femoral neck fractures and left sided avascular necrosis, treated subsequently with bilateral total hip replacement. Histological analysis confirmed the presence of a phosphaturic mesenchymal tumor in the left femoral head measuring $27 \mathrm{~mm}$. Post-operative FGF23 declined to $12.7 \mathrm{pg} / \mathrm{mL}$ post-operatively and remained within the normal reference at $43.1 \mathrm{pg} / \mathrm{mL}$ at 10 months after the surgery. His recovery was complicated by "Hungry Bone Syndrome" treated successfully with calcium and calcitriol replacement. He reported complete resolution of his chronic pain. He has become more independent with the activities of daily living without falls or fractures, at 10 months post-surgery. He no longer suffers from severe depression, and he has actively participated in his rehabilitation program. Mark's progress was assessed by the 36-Item Short Form Health Survey (SF-36) with a $0-100$ scale. The score of 0 in SF-36 is indicative of the maximum disability while a score of 100 is equivalent to no disability (Ware and Sherbourne 1992). Postoperative improvement in patient's health status [quality of life (QoL) score of 80 out of 100)] was reflected by his highly perceived satisfaction with change in his overall health (QoL score of 100). In particular, the patient reported a major improvement in his social functioning (QoL score of 50) with lesser change (QoL scores of 30) for both his physical functioning and emotional well-being.

\section{Discussion}

The present case illustrates the prolonged and dramatic presentation of a patient with TIO that significantly affected all aspects of patient's life. $\mathrm{He}$ presented with bone pain, muscle weakness, and pathological fractures, in the context of severely low bone density, which was initially attributed to hypogonadism (Campion and Maricic 2003). Delayed diagnosis of TIO severely affected his physical health leaving him bed bound with profound depression subsequently leading to adverse financial outcomes. TIO has been previously reported to exert negative psychological impact on affected individuals due profound physical disability, severe pain, and prolonged time leading to diagnosis, in more extreme cases culminating in patient's suicidal attempts (Seemann et al. 2019). In particular, TIO can have devastating impact on psychological wellbeing in patients, who are affected by an underlying mental illness. Nonspecific symptoms of TIO may lead to erroneous diagnoses of other rheumatological, endocrinological or neurological disorders.

Classic features of osteomalacia of any cause include bone pain, musculoskeletal weakness, and recurrent pathological fractures (Econs and Drezner 1994). Laboratory features of TIO include hypophosphatemia, phosphaturia, normal PTH and serum calcium, decreased $1,25(\mathrm{OH})_{2} \mathrm{D}$, elevated ALP and raised FGF23 (Jan de Beur 2005; Wu et al. 2017; Yu et al. 2017). Differential diagnosis includes hereditary hypophosphatemic syndromes, and clues to these include short stature, rachitic bony deformity and poor dentition; genetic testing for PHEX (XLH), FGF23 (ADHR), and DMP1 or ENPP1 (ARHR) may be useful in excluding these disorders (The HYP Consortium 1995; ADHR Consortium 2000).

TIO tumors are divided into 4 subtypes: phosphaturic mesenchymal tumor (PMT) mixed connective tissue variant, osteoblastoma-like variant, ossified fibroid-like variant, and non-ossifying fibroma-like variant, with PMT being the most common (Econs and Drezner 1994; Wu et al. 2017). They are generally located in bone or soft tissues (Siegel et al. 2002).

Once diagnosed, TIO prognosis is generally good as most FGF23-secreting tumors are benign and rarely metastasize (Edmister and Sundaram 2002). However, diagnosis of these tumors is frequently delayed by many years due to their occult nature, small size and atypical location (Jagtap et al. 2011). Tumor resection leads to prompt correction of all the clinical and biochemical manifestations of this syndrome (Ryan and Reiss 1984; Chong et al. 2011), 
and gradual re-mineralization of the osteoid matrix (Hautmann et al. 2015). Localization of FGF23 secreting tumors is often challenging as they are often small in size and slow growing, moreover, they are frequently located in atypical skeletal sites (Liu et al. 2019). Various imaging techniques are often used including functional imaging with octreotide scintigraphy, fluorodeoxyglucose (FDG) PET/CT, ${ }^{68} \mathrm{Ga}$ DOTATATE PET/CT, anatomical imaging with MRI and selective venous sampling for FGF23 levels (Colt et al. 2005; Clifton-Bligh et al. 2013; El-Maouche et al. 2016). In our case, ${ }^{68} \mathrm{Ga}$ DOTATATE PET/CT detected the tumor lesion in our patient. The high sensitivity and specificity of ${ }^{68} \mathrm{Ga}$ DOTATATE PET/CT favors its early use in suspected cases of TIO (Clifton-Bligh et al. 2013).

Medical treatment of TIO involves high doses of oral phosphate and calcitriol, as oral phosphate replacement alone is usually insufficient for correcting hypophosphatemia. Gastrointestinal absorption phosphate remains impaired in the setting of low $1,25(\mathrm{OH})_{2} \mathrm{D}$ (Slot-Steenks et al. 2016). Oral phosphate replacement is often poorly tolerated and leads to diarrhea with subsequent noncompliance.

In summary, our case illustrates an insidious and dramatic impact of TIO on physical health, psychological and financial aspects of life in the person who is affected by this debilitating medical condition. Many factors contributed to delayed diagnosis of TIO in this case, including his physical disability with limited access to medical care, lack of awareness of this rare condition among clinicians and restricted diagnostic resources. The present case highlights the need to extensively investigate the causes of generalized bone pain in patients with hypophosphatemia, as TIO is highly curable (Siegel et al. 2002). Furthermore, prompt diagnosis and tumor excision can result in dramatic an improvement in quality of life.

\section{References}

ADHR Consortium. Autosomal dominant hypophosphataemic rickets is associated with mutations in FGF23. Nat Genet 26, 345-348, 2000.

Campion J, Maricic M. Osteoporosis in men. Am Fam Physician 67, 1521-1526, 2003.

Carpenter T. Oncogenic osteomalacia--a complex dance of factors. N Engl J Med 348, 1705-1708, 2003.

Chong W, Molinolo A, Chen C, Collins M. Tumor-induced osteomalacia. Endocr Relat Cancer 18, R53-77, 2011.

Clifton-Bligh R, Hofman M, Duncan E, Sim Ie, Darnell D, Clarkson A, Wong T, Walsh J, Gill A, Ebeling P, Hicks R. Improving diagnosis of tumor-induced osteomalacia with Gallium-68 DOTATATE PET/CT. J Clin Endocrinol Metab 98, 687-694, 2013.

Colt E, Gopan T, Chong H. Oncogenic osteomalacia cured by removal of an organized hematoma. Endocrine Practice 11, 190-193, 2005.

Econs M, Drezner M. Tumor-induced osteomalacia--unveiling a new hormone. N Engl J Med 330, 1679-1681, 1994.

Edmister K, Sundaram M. Oncogenic osteomalacia. Semin Musculoskelet Radiol 6, 191-196, 2002.

El-Maouche D, Sadowski S, Papadakis G, Guthrie L, Cottle-Delisle C, Merkel R, Millo C, Chen C, Kebebew E, Collins MT. (68)Ga-DOTATATE for tumor localization in tumor-induced osteomalacia. J Clin Endocrinol Metab 101, 3575-3581, 2016.

Hautmann A, Hautmann M, Kolbl O, Herr W, Fleck M. Tumor-induced osteomalacia: an up-to-date review. Curr Rheumatol Rep 17, 512, 2015.

Hu F, Jiang C, Zhang Q, Shi H, Wei L, Wang Y. Quantitative ELISA-like immunohistochemistry of fibroblast growth factor 23 in diagnosis of tumor-induced osteomalacia and clinical characteristics of the disease. Dis Markers 2016, 3176978, 2016.

Jagtap V, Sarathi V, Lila A, Malhotra G, Sankhe S, Bandgar T, Menon P, Shah N. Tumor-induced osteomalacia: a single center experience. Endocrine Practice 17, 177-184, 2011.

Jan de Beur SM. Tumor-induced osteomalacia. JAMA 294, 1260-1267, 2005.

Jonsson K, Zahradnik R, Larsson T, White K, Sugimoto T, Imanishi Y, Jamamoto T, Hampson G, Koshiyama H, Ljunggren O, Oba K, Yang M, Miyauchi A, Econs M, Lavinge J, Juppner H. Fibroblast growth factor 23 in oncogenic osteomalacia and X-linked hypophosphatemia. N Engl J Med 348, 1656-1663, 2003.

Liu S, Zhou X, Song A, Huo Z, Wang Y, Xia W, Liu Y. Successful treatment of tumor-induced osteomalacia causing by phosphaturic mesenchymal tumor of the foot. Medicine (Baltimore) 98, e16296, 2019.

Perwad F, Zhang M, Tenenhouse H, Portale A. Fibroblast growth factor 23 impairs phosphorus and vitamin D metabolism in vivo and suppresses 25-hydroxyvitamin D-1alpha-hydroxylase expression in vitro. Am J Physiol Renal Physiol 293, F1577-F1583, 2007. 
Ryan E, Reiss E. Oncogenous osteomalacia. Review of the world literature of 42 cases and report of two new cases. Am J Med 77, 501-512, 1984.

Seemann L, Padala S, Mohammed A, Belayneh N. Tumor-induced osteomalacia and the importance of plasma fibroblast growth factor 23 as an indicator: diagnostic delay leads to a suicide attempt. J Investig Med High Impact Case Rep 7, 2324709619895162, 2019.

Siegel H, Rock M, Inwards C, Sim F. Phosphaturic mesenchymal tumor. Orthopedics 25, 1279-1281, 2002.

Slot-Steenks M, Hamdy N, van de Sande M, Vriens D, Cleven A, Appelman-Dijkstra N. Identifying the culprit lesion in tumor induced hypophosphatemia, the solution of a clinical enigma. Endocrine 54, 642-647, 2016.

The HYP Consortium. A gene (PEX) with homologies to endopeptidases is mutated in patients with X-linked hypophosphatemic rickets. Nat Genet 11, 130-136, 1995.

Ware JE Jr, Sherbourne CD. The MOS 36-item short-form health survey (SF-36). I. Conceptual framework and item selection. Medical Care 30, 473-483, 1992.

Wu W, Wang C, Ruan J, Chen F, Li N, Chen F. A case report of phosphaturic mesenchymal tumor-induced osteomalacia. Medicine (Baltimore) 96, e9470. 2017.

Yamazaki Y, Okazaki R, Shibata M, Hasegawa Y, Satoh K, Tajima T, Takeuchi Y, Fujita T, Nakahara K, Yamashita T, Fukumoto S. Increased circulatory level of biologically active full-length FGF-23 in patients with hypophosphatemic rickets/osteomalacia. J Clin Endocrinol Metab 87, 4957-4960, 2002.

$\mathrm{Yu}$ W, He J, Fu W, Wang C, Zhang Z. Reports of 17 Chinese patients with tumor-induced osteomalacia. J Bone Miner Metab 35, 298-307, 2017. 